\title{
Bayesian Data Fusion of Multiview Synthetic Aperture Sonar Imagery for Seabed Classification
}

\author{
David P. Williams
}

\begin{abstract}
A Bayesian data fusion approach for seabed classification using multiview synthetic aperture sonar (SAS) imagery is proposed. The principled approach exploits all available information and results in probabilistic predictions. Each data point, corresponding to a unique $10 \mathrm{~m} \times 10 \mathrm{~m}$ area of seabed, is represented by a vector of wavelet-based features. For each seabed type, the distribution of these features is then modeled by a unique Gaussian mixture model. When multiple views of the same data point (i.e., area of seabed) are available, the views are combined via a joint likelihood calculation. The end result of this Bayesian formulation is the posterior probability that a given data point belongs to each seabed type. It is also shown how these posterior probabilities can be exploited in a form of entropy-based active-learning to determine the most useful additional data to acquire. Experimental results of the proposed multiview classification framework are shown on a large data set of real, multiview SAS imagery spanning more than $2 \mathbf{k m}^{2}$ of seabed.
\end{abstract}

Index Terms-Bayesian data fusion, Gaussian mixture models (GMMs), multiview data, seabed classification, seabed segmentation, synthetic aperture sonar (SAS).

\section{INTRODUCTION}

$\mathbf{S}$ EABED classification is the process by which one segments a typically large area of seabed into different regions based on the characteristics of the seabed. For example, an area may be segmented into flat seabed, rocky seabed, and seabed characterized by sand ripples. The need to perform this seabed classification in an automated manner with no human intervention is motivated by the desire to conduct fully autonomous mine countermeasures (MCM) missions with an autonomous underwater vehicle (AUV).

Most MCM AUV missions are either clearance missions or route-finding missions. In a clearance mission, the objective is to detect, classify, and clear mines from an area. Route-finding missions, in contrast, attempt to find low-risk routes that can be used safely by subsequent assets. In both scenarios, establishing the seabed type in the area is important because mine detection and classification performance is a strong function of seabed type [1]. For example, because attempting to detect a mine in a

Manuscript received October 13, 2008; revised February 06, 2008. First published May 02, 2009; current version published May 13, 2009. The associate editor coordinating the review of this manuscript and approving it for publication was Prof. Scott T. Acton.

The author is with the NATO Undersea Research Centre, 19126 La Spezia (SP), Italy (e-mail: williams@ @urc.nato.int).

Color versions of one or more of the figures in this paper are available online at http://ieeexplore.ieee.org.

Digital Object Identifier 10.1109/TIP.2009.2017161 boulder field is prohibitively difficult, routes that pass through rocky areas of seabed would be deemed to be unfeasible.

A fundamental justification for employing intelligent autonomy systems for MCM missions is the assumption that the classification of an area of seabed-or of an object-will improve as the number of views of it increases. For instance, if a mine is observed from one orientation, it may be difficult to distinguish it from a rock; however, viewing it from a second orientation may reveal previously obscured characteristics that differentiate it from a rock.

The use of AUVs that can adapt their routes is closely related to the topic of active learning. Generally speaking, active learning is concerned with intelligently selecting which additional data to acquire in order to most improve classification [2]. Active learning techniques have been applied to machine learning problems, but never in the context of seabed classification.

\section{A. Previous Seabed Classification Approaches and Motivation for Proposed Approach}

The earliest approaches to the seabed classification problem focused on devising features that would be able to discriminate the various seabed types, but considered only single images [3]-[7]. Later work [8]-[10] began to address the problem under the assumption that the seabed classification was to be performed on multiple, partially overlapping images. More specifically, those approaches focused on the manner in which separate classification decisions made from individual images would be fused into a single unified decision. That is, a standard single-view seabed classification approach was adopted, with fusion of the classification decisions performed subsequently.

Because a principled foundational framework for directly addressing the multi-view case was not used, various ad hoc compromises were needed to obtain the final classification. This type of fusion approach then forced the final classifications to be hard decisions rather than probabilistic predictions. However, the classification of seabed type is rarely a simple clear-cut decision.

An example that illustrates this fact (and which highlights the advantage of probabilistic predictions) is shown in Fig. 1.

This figure shows a $10 \mathrm{~m} \times 10 \mathrm{~m}$ area of seabed-which corresponds to a single data point in this paper-that contains characteristics of both flat and rippled seabeds. In cases such as this, forcing a hard decision regarding seabed type when one is not justified ignores the full complexity of the seabed classification problem.

Moreover, because the seabed type predictions of the previous approaches were hard decisions rather than probabilistic 


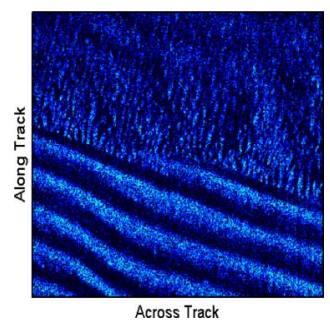

Fig. 1. Example synthetic aperture sonar image (data point) for which a hard decision regarding seabed type is inappropriate.

in nature, the benefit of viewing an area of seabed multiple times was not quantifiable. That is, there would be no way (during the experiment) to ascertain whether viewing an area of seabed one time was any more beneficial than viewing it two times; in either case, the seabed prediction would be a simple hard decision.

In contrast, with probabilistic predictions, one can quantify the amount by which the seabed-type prediction could improve if an additional view was obtained. In this manner, when faced with limited resources, one can establish which areas of the seabed would most greatly benefit from additional views, and which areas are already classified to a sufficiently high level of confidence from a single view.

Finally, the use of a Bayesian classification approach would provide a principled way to leverage prior knowledge possessed about the seabed classification task with observations (i.e., data).

Because of these reasons, it is imperative that a principled Bayesian multiview seabed classification algorithm that results in probabilistic predictions be employed. The proposed algorithm exploits these considerations in its formulation.

\section{B. Contribution of Paper}

The seabed classification approach proposed in this paper is a principled Bayesian data fusion method that exploits all available information. Each data point-corresponding to a $10 \mathrm{~m} \times 10 \mathrm{~m}$ area of seabed in this paper-is represented by a vector of wavelet-based features. For each seabed type, the distribution of these features is then modeled by a unique Gaussian mixture model. When multiple views of the same data point (i.e., area of seabed) are available, the views are combined via a joint likelihood calculation. The end result of this Bayesian formulation for a given data point is the posterior probability that it belongs to each seabed type. These posterior probabilities can then be used both to classify the seabed probabilistically, and also to guide active learning.

The main contribution of this paper is the development of a fully Bayesian framework that fuses data from multiple views in a principled manner to perform seabed classification. Importantly, the probabilistic nature of the classification predictions also allows an appealingly intuitive approach to perform active learning. Specifically, the uncertainty of the classification predictions, as quantified by the entropy, is used to determine the areas of seabed for which the acquisition of additional views would most improve classification confidence.

Whereas all previous seabed classification work has considered simple side-scan sonar imagery, this paper is the first to address the task when using high-resolution synthetic aperture sonar (SAS) imagery. Furthermore, the idea of performing active learning to decide which additional data to collect has never before been broached in this context of seabed classification.

\section{Structure of Paper}

The remainder of this paper is organized in the following manner. In Section II, Gaussian mixture models (GMMs) are discussed. The manner in which these GMMs are employed to perform Bayesian multiview data fusion is explained in Section III. The application of active learning to the multiview classification problem is discussed in Section IV. Section V describes the real, multiview, SAS imagery data set on which the proposed seabed classification approach is applied. The experimental set-up is outlined in Section VI, with classification and active-learning results shown in Section VII. The proposed approach is discussed in Section VIII before concluding remarks are made in Section IX.

\section{Gaussian MiXture Models (GMMS)}

It is well-known that a Gaussian mixture model (GMM) can accurately model an arbitrary continuous distribution. Moreover, this model estimation can be performed efficiently using the expectation-maximization (EM) algorithm [11] or the variational Bayesian EM (VB-EM) algorithm [12]. Whereas the result of the EM algorithm will be point estimates for the parameters (i.e., the mixing proportions, means, and covariances), the result of the VB-EM algorithm will be full distributions of the parameters. This fact allows the GMM learned via the VB-EM algorithm to be accurate even when faced with limited data.

With an EM approach, one must specify the number of mixture components a priori. In contrast, the VB-EM approach will automatically infer the appropriate number of mixture components that are represented by the data [13], [14]. Whereas the standard EM algorithm will always favor increasingly complex models (i.e., more mixture components), the variational formulation automatically penalizes overly complex models. For these reasons, we choose to employ the VB-EM algorithm for estimating the requisite GMMs in this paper.

In particular, the distribution of features of each seabed type is modeled with its own unique GMM. Therefore, for $J$ seabed types, $J$ separate GMMs will be learned.

This supervised approach of course requires that labeled training data is available to perform the estimation. One alternative approach would instead learn a single GMM for all seabed types jointly. In this case, no labeled training data would be required. However, with this unsupervised approach, each seabed type would most likely be represented by multiple Gaussians of the GMM (because a single Gaussian would not be able to capture the full variability and complexity of a given seabed type); human intervention would then be required to establish the correspondence between seabed types and Gaussians. In the supervised approach we employ, the mapping from Gaussians to seabed types is straightforward because each seabed type is associated with its own GMM; hence, no human assistance is required.

In this paper, we model the distribution of the $d$-dimensional feature vector, $\boldsymbol{x}$, extracted from an area of seabed — conditioned 
on each seabed type-with a GMM. The GMM corresponding to seabed type $s_{j}$ is

$$
\begin{aligned}
p\left(\boldsymbol{x} \mid s_{j}\right) & \\
= & \sum_{k=1}^{K_{j}} \alpha_{k}^{j}(2 \pi)^{-d / 2}\left|\boldsymbol{\Sigma}_{k}^{j}\right|^{-1 / 2} \\
& \quad \times \exp \left\{-\frac{1}{2}\left(\boldsymbol{x}-\boldsymbol{\mu}_{k}^{j}\right)^{T}\left(\boldsymbol{\Sigma}_{k}^{j}\right)^{-1}\left(\boldsymbol{x}-\boldsymbol{\mu}_{k}^{j}\right)\right\} \\
& \equiv \sum_{k=1}^{K_{j}} \alpha_{k}^{j} \mathcal{N}\left(\boldsymbol{x} ; \boldsymbol{\mu}_{k}^{j}, \boldsymbol{\Sigma}_{k}^{j}\right)
\end{aligned}
$$

where $\alpha_{k}^{j}, \boldsymbol{\mu}_{k}^{j}$, and $\boldsymbol{\Sigma}_{k}^{j}$ are the mixing proportion, mean, and covariance, respectively, of the $k$ th Gaussian of the $K_{j}$-component GMM corresponding to seabed type $s_{j}$. The non-negative mixing proportions satisfy $\sum_{k=1}^{K_{j}} \alpha_{k}^{j}=1$.

\section{BAYESIAN MULTIVIEW DATA Fusion}

\section{A. General Case}

When a data point (i.e., area of seabed), $\boldsymbol{x}_{i}$, is seen a single time, the likelihood that the data point is seabed type $s_{j}$ is given by the GMM corresponding to $s_{j}$

$$
p\left(\boldsymbol{x}_{i} \mid s_{j}\right)=\sum_{k=1}^{K_{j}} \alpha_{k}^{j} \mathcal{N}\left(\boldsymbol{x}_{i} ; \boldsymbol{\mu}_{k}^{j}, \boldsymbol{\Sigma}_{k}^{j}\right) .
$$

By Bayes' rule, the posterior probability that the data point is seabed type $s_{j}$ is then

$$
p\left(s_{j} \mid \boldsymbol{x}_{i}\right)=\frac{p\left(\boldsymbol{x}_{i} \mid s_{j}\right) p\left(s_{j}\right)}{\sum_{j=1}^{J} p\left(\boldsymbol{x}_{i} \mid s_{j}\right) p\left(s_{j}\right)}
$$

where $p\left(s_{j}\right)$ is the prior probability that a data point is seabed type $s_{j}$ out of $J$ possible seabed types. If no a priori knowledge is available, uninformative (i.e., equal) priors can be specified for each seabed type. The denominator in (4) is simply a normalizing factor, called the evidence.

Now suppose a data point (i.e., area of seabed) is seen two times, with these two views represented by $\boldsymbol{x}_{i}^{1}$ and $\boldsymbol{x}_{i}^{2}$. Assuming the views are independent, the likelihood that the data point is seabed type $s_{j}$ becomes

$$
\begin{aligned}
p\left(\boldsymbol{x}_{i}^{1}, \boldsymbol{x}_{i}^{2} \mid s_{j}\right)= & p\left(\boldsymbol{x}_{i}^{1} \mid s_{j}\right) p\left(\boldsymbol{x}_{i}^{2} \mid s_{j}\right) \\
= & {\left[\sum_{k=1}^{K_{j}} \alpha_{k}^{j} \mathcal{N}\left(\boldsymbol{x}_{i}^{1} ; \boldsymbol{\mu}_{k}^{j}, \boldsymbol{\Sigma}_{k}^{j}\right)\right] } \\
& \times\left[\sum_{\ell=1}^{K_{j}} \alpha_{\ell}^{j} \mathcal{N}\left(\boldsymbol{x}_{i}^{2} ; \boldsymbol{\mu}_{\ell}^{j}, \boldsymbol{\Sigma}_{\ell}^{j}\right)\right] .
\end{aligned}
$$

The posterior probability that the data point is seabed type $s_{j}$ is then simply

$$
p\left(s_{j} \mid \boldsymbol{x}_{i}^{1}, \boldsymbol{x}_{i}^{2}\right)=\frac{p\left(\boldsymbol{x}_{i}^{1}, \boldsymbol{x}_{i}^{2} \mid s_{j}\right) p\left(s_{j}\right)}{\sum_{j=1}^{J} p\left(\boldsymbol{x}_{i}^{1}, \boldsymbol{x}_{i}^{2} \mid s_{j}\right) p\left(s_{j}\right)} .
$$

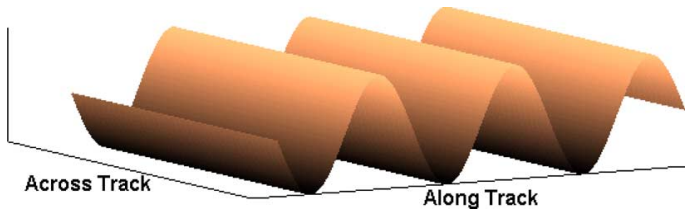

Fig. 2. Seabed characterized by sand ripples will appear to be flat seabed when the sand ripple "waves" propagate in the along-track direction of the vehicle.

For a data point (i.e., area of seabed), $\boldsymbol{x}_{i}$, that is seen $V_{i}$ times, let its views be represented compactly by $\mathbf{X}_{i}=\left\{\boldsymbol{x}_{i}^{1}, \boldsymbol{x}_{i}^{2}, \cdots, \boldsymbol{x}_{i}^{V_{i}}\right\}$. For this general case of $V_{i}$ (again, assumed independent) views, the likelihood that the data point is seabed type $s_{j}$ is

$$
\begin{aligned}
p\left(\mathbf{X}_{i} \mid s_{j}\right) & =p\left(\boldsymbol{x}_{i}^{1} \mid s_{j}\right) p\left(\boldsymbol{x}_{i}^{2} \mid s_{j}\right) \cdots p\left(\boldsymbol{x}_{i}^{V_{i}} \mid s_{j}\right) \\
& =\prod_{v=1}^{V_{i}} p\left(\boldsymbol{x}_{i}^{v} \mid s_{j}\right) \\
& =\prod_{v=1}^{V_{i}} \sum_{k=1}^{K_{j}} \alpha_{k}^{j} \mathcal{N}\left(\boldsymbol{x}_{i}^{v} ; \boldsymbol{\mu}_{k}^{j}, \boldsymbol{\Sigma}_{k}^{j}\right) .
\end{aligned}
$$

The posterior probability that the data point is seabed type $s_{j}$ is then

$$
p\left(s_{j} \mid \mathbf{X}_{i}\right)=\frac{p\left(\mathbf{X}_{i} \mid s_{j}\right) p\left(s_{j}\right)}{\sum_{j=1}^{J} p\left(\mathbf{X}_{i} \mid s_{j}\right) p\left(s_{j}\right)} .
$$

The independence assumption for multiple views is not completely justified because each view would be observing the same general area of seabed. However, each data point is not a single location on the seabed, but is rather an area of seabed (with spatial extent). As a result, when viewing a data point from different directions, not all of the same physical seabed locations will constitute the data point. Moreover, the fact that objects on the seabed (such as rocks or mines) and seabed characteristics (like sand ripples) can appear very different from different orientations should also ameliorate this assumption.

\section{B. Special Case: Orientation Dependence of Sand Ripples}

The posterior probability that a data point is a particular seabed type, based on multiple views, was given in (11). Implicit in its derivation, however, was that a given area of seabed would always appear as the true seabed type from any orientation. For example, a rocky seabed would look like a rocky seabed regardless of the orientation from which it was viewed. This assumption is not always true, however. In particular, seabed characterized by sand ripples exhibits a strong orientation dependence; when the sand ripple "waves" propagate in the along-track direction of the vehicle (which is assumed to be orthogonal to the look-direction of the sonar), the seabed appears to be flat. A cartoon depicting this scenario is shown in Fig. 2, while real SAS imagery illustrating this phenomenon is shown in Fig. 3.

To account for this orientation dependence of ripples, the likelihood in (9) for the multiview case must be modified slightly. Without loss of generality, let $s_{1}$ and $s_{2}$ denote flat seabed and rippled seabed, respectively. 


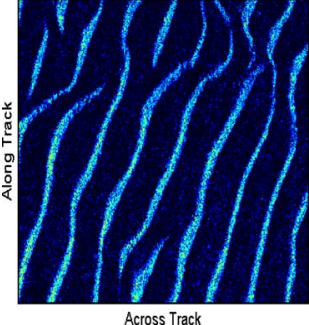

(a)

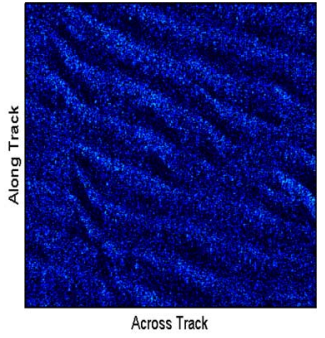

(b)
Fig. 3. SAS imagery of the same area of seabed characterized by ripples when the along-track direction of the vehicle is (a) nearly perpendicular to the ripple propagation direction and (b) nearly parallel to the ripple propagation direction.

Let $\theta_{\ell}$ denote the orientation of the $\ell$ th of $V_{i}$ views. Assume that the orientations of the $V_{i}$ views are sufficiently separated such that $\left|\theta_{a}-\theta_{b}\right|>\delta$ for all pairs of views, where $\delta \in$ $\left[0^{\circ}, 180^{\circ}\right]$ is the assumed span of orientations over which a rippled seabed will appear to be flat. Also define $\lambda=\delta / 180^{\circ}$.

For the case of $V_{i}$ views, the likelihood of the data point being rippled seabed, $s_{2}$, is modified to be

$$
\begin{aligned}
p^{\prime}\left(\mathbf{X}_{i} \mid s_{2}\right)=(1-\lambda) & \prod_{v=1}^{V_{i}} p\left(\boldsymbol{x}_{i}^{v} \mid s_{2}\right) \\
& +\lambda \sum_{u=1}^{V_{i}} p\left(\boldsymbol{x}_{i}^{u} \mid s_{1}\right) \prod_{w \neq u} p\left(\boldsymbol{x}_{i}^{w} \mid s_{2}\right)
\end{aligned}
$$

where $p\left(\boldsymbol{x}_{i} \mid s_{j}\right)$ is as defined in (3). The modification in (12) allows for the case in which one of the views of a rippled seabed appears to be flat seabed. As $\lambda \rightarrow 0$, which corresponds to the case in which a rippled seabed never appears to be flat seabed, (12) degenerates into (9).

The assumption that the orientations of the views are sufficiently separated enforces that no more than one view of rippled seabed appears to be flat seabed. This assumption is justified by the fact that in practice, a sonar-equipped vehicle will be programmed to take routes that maximize the angular diversity among orientations (so that the most informative data can be collected). Although this assumption is not imperative, without it the resulting equation would be more cumbersome and less general (with a greater dependence on the specific orientations taken).

\section{ENTROPY-BASED ACTIVE LEARNING}

Active learning deals with the intelligent acquisition of additional data to improve classification [2]. Traditionally the topic of active learning is considered in the context of acquiring the label (e.g., mine or clutter) of an object in classification tasks. But it is equally valid to consider the additional data to be in the form of additional views from a given sensor or views from a new sensor [15].

Active learning seeks to answer the following question: If it is possible to acquire additional data, which data should be collected to most improve classification? Interestingly, this fundamental statement of active learning mirrors the very motivation for employing autonomous underwater vehicles. Namely, the purpose of giving an AUV the freedom to adapt its route in-mission is to allow it to collect the most useful data.

In the context of seabed classification, the logical approach for active learning is to acquire additional data (i.e., views) for the areas of seabed for which the current classifications have the highest uncertainty. In this paper, we quantify this uncertainty via the entropy of the probabilistic classification predictions.

The entropy (in bits) of a discrete random variable, $X$, is defined as [16]

$$
H(X)=-\sum_{x \in \chi} p(x) \log _{2} p(x)
$$

where $p(x)$ is the probability that $X$ takes on the value $x$ in the alphabet $\chi$ of possible values.

If the seabed type of a data point, $\mathbf{X}_{i}$, is treated as a random variable, the entropy (in bits) of the seabed-type prediction in terms of the posterior probabilities, $p\left(\mathbf{X}_{i} \mid s_{j}\right)$, can be written as

$$
H\left(\mathbf{X}_{i}\right)=-\sum_{j=1}^{J} p\left(\mathbf{X}_{i} \mid s_{j}\right) \log _{2} p\left(\mathbf{X}_{i} \mid s_{j}\right)
$$

where it is assumed that there are $J$ possible seabed types.

By employing this entropy-based criterion for active learning, the resources for collecting the additional data would be optimally allocated for improving the confidence in the classification task.

It should be emphasized that without the principled probabilistic foundations of the proposed multiview classification approach, such an elegantly simple active-learning method would not be possible. It is the fully probabilistic nature of the proposed multiview classification framework that allows such an intuitive approach to active learning to fall out naturally.

\section{DATA SET}

\section{A. Synthetic Aperture Sonar (SAS) Imagery}

In April-May 2008, the NATO Undersea Research Centre (NURC) conducted the Colossus II sea trial in the Baltic Sea off the coast of Latvia. During this trial, high-resolution sonar data was collected by the MUSCLE autonomous underwater vehicle (AUV). This AUV is equipped with a $300 \mathrm{kHz}$ sonar with a $60 \mathrm{kHz}$ bandwidth that can achieve image resolution of approximately $3 \mathrm{~cm}$. From April 20-22, the vehicle collected data over an approximately $2 \mathrm{~km}^{2}$ area in four different survey directions (north-south, northeast-southwest, east-west, and southeast-northwest). The track spacing of the vehicle was designed to prevent dead-zones (lacking sonar coverage), assuming sonar coverage would be achieved from 40 to $150 \mathrm{~m}$ on either side of the vehicle. The routes of the vehicle in each survey direction are shown in Fig. 4.

The enormous amount of data that resulted from this collection was then processed into synthetic aperture sonar (SAS) imagery. This particular three-day data set contains 3550 SAS images (each covering an area of $30 \mathrm{~m} \times 110 \mathrm{~m}$ ), one example of which is shown in Fig. 5.

In this paper, the "atomic" unit for seabed classification is assumed to be a $10 \mathrm{~m} \times 10 \mathrm{~m}$ block of seabed. That is, each $10 \mathrm{~m} \times 10 \mathrm{~m}$ block of seabed corresponds to one data point. 


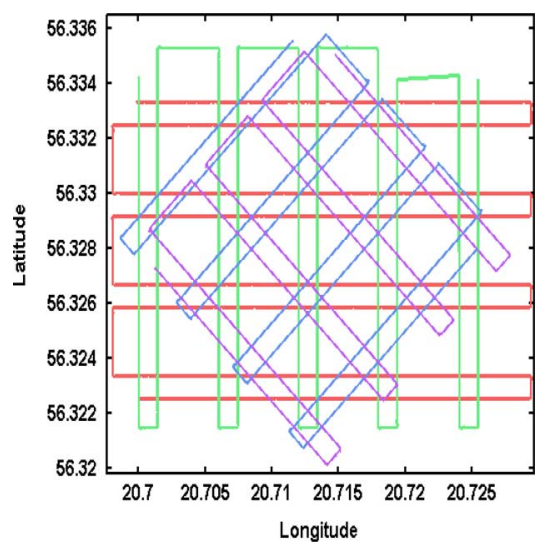

Fig. 4. Routes of the AUV, with each survey direction shown in a different color, for the data set.

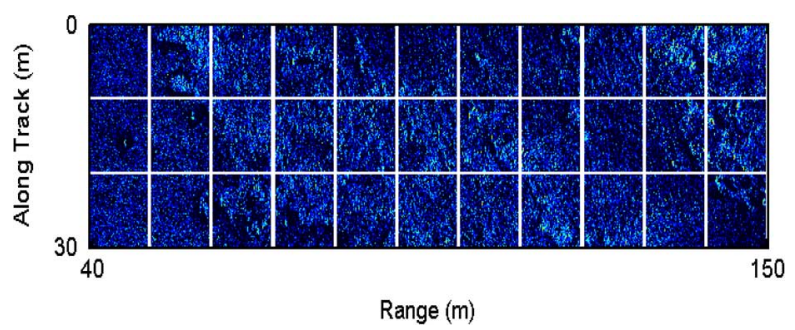

Fig. 5. Example SAS image, with $10 \mathrm{~m} \times 10 \mathrm{~m}$ blocks of seabed demarcated by the overlaid white lines.

This particular size was chosen as a compromise among several factors. The larger the area of a block, the more likely that a single block will have the unfavorable property of containing multiple types of seabed. However, if the block area is too small, the distinguishing characteristics of the seabed that indicate a certain seabed type may be lost. Moreover, the subsequent uses of the seabed classification results (such as AUV path-planning for MCM operations) do not require seabed classification at excessively fine resolution.

Each data point-a $10 \mathrm{~m} \times 10 \mathrm{~m}$ block of seabed-is in turn represented by a vector of features, $\boldsymbol{x}$. Our objective is to classify the seabed type that each data point represents, based on its features. The features should, therefore, be able to discriminate among different seabed types.

In this paper, we employ a set of 16 features that are derived from the coefficients of a biorthogonal wavelet decomposition [17] of the SAS image block. Specifically, each feature of a block corresponds to the quadratic mean (i.e., rootmean-square (RMS) value) of the block's wavelet coefficient amplitudes at a particular orientation and scale. This particular set of features was chosen because it can successfully capture the distinguishing textural properties of the seabed. Namely, the wavelet-coefficient energy will be large when the orientation and scale match the orientation and scale of high-energy texture components in the image block [17].

However, it should be emphasized that the proposed seabed classification algorithm is general in the sense that any reasonable set of features can be employed. The main focus of this paper is not the development of a new set of features for seabed

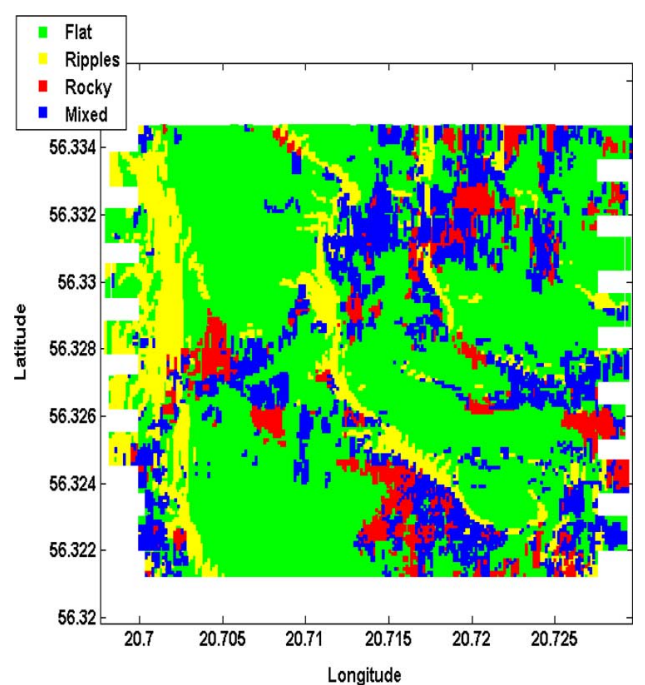

Fig. 6. Manual ground-truth of the data from the east-west survey direction. Each pixel corresponds to a $10 \mathrm{~m} \times 10 \mathrm{~m}$ area of seabed.

classification, but rather is a proof-of-concept of the proposed multiview fusion approach.

\section{B. Ground Truth}

The objective in the ensuing experiments is to accurately classify the seabed. To this end, all of the data (i.e., SAS image blocks of seabed) from the east-west survey direction were painstakingly ground-truthed ("manually," by visual inspection) as belonging to one of four seabed types. The seabed types considered were: flat seabed, rippled seabed, rocky seabed, and mixed seabed. The "mixed seabed" class is a potpourri of seabed blocks for which classification as one of the other three classes was not appropriate. This class is often characterized by flat seabed with shell deposits (whereas the "flat seabed" class is usually characterized by a softer, mud-like composition free of such deposits). The ground-truth of the east-west survey direction is shown in Fig. 6.

The manual ground-truthing is a subjective, inherently imprecise process; for example, multiple seabed types can exist within a single block (which nevertheless must be classified as a single seabed type). The general difficulty of the groundtruthing task can be realized if one attempts to manually groundtruth the example SAS image shown in Fig. 5. It should be noted, therefore, that obtaining a seabed classification result that perfectly matches the manual ground-truth is an unrealistic - and not completely desirable—-goal.

Nevertheless, to provide rough evidence that the manual ground-truth is plausibly accurate enough to be used for the seabed classification task, we show the result of a (contact) detection algorithm over the same area. The (contact) detection algorithm [18] employs a matched filter to find highlight-shadow patterns characteristic of mine-like objects in the images. The result of the detection algorithm on the SAS imagery from each of the four survey directions is shown in Fig. 7.

The general correspondence between the manual groundtruth map in Fig. 6 and the contact-detection map in Fig. 7 suggests that the ground-truth result is reasonable (e.g., 


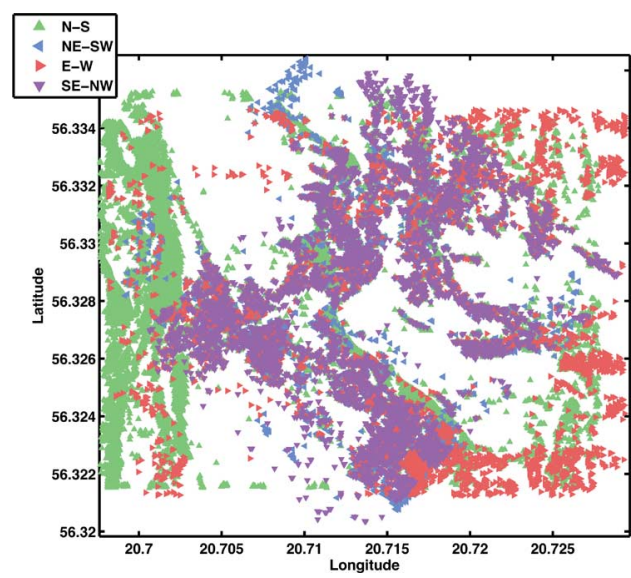

Fig. 7. Contacts detected. Each point corresponds to one detection, with color used to distinguish the survey direction from which each detection was made.

areas ground-truthed as flat are typically free of detections, while areas ground-truthed as rocky typically contain many detections).

\section{EXPERIMENTAL SET-UP}

\section{A. Training Procedure}

Associated with each $10 \mathrm{~m} \times 10 \mathrm{~m}$ block of seabed are latitude and longitude coordinates, corresponding to the center of the block. If the distance between the center points of two blocks on different survey directions is below a set threshold, we consider the two blocks to correspond to the same data point. It is in this manner that a data point can be viewed multiple times. As noted previously, however, the multiple views corresponding to a given data point will necessarily be comprised of nonidentical physical seabed locations.

In Section III-B, it was discussed that seabed characterized by sand ripples exhibits a special orientation dependence. When the along-track direction of the vehicle is similar to the propagation direction of the ripples, the rippled seabed can appear to be flat seabed. For the results presented here, it is assumed that there exists a $\delta=20^{\circ}$ span of angles in which rippled seabed appears to be flat, so $\lambda=1 / 9$ in (12).

Because a supervised approach is employed to learn the GMM for each seabed type, some labeled data must be available for the training procedure. In this paper, we learn the requisite GMMs using those seabed blocks from the east-west survey (for which manual ground-truthing has been performed) of data points that have no more than one additional view from the other survey directions. (Each data point in the data set has between one and four views, because the four surveys do not cover identical areas. Thus, to learn the GMMs, only views from the east-west survey of certain data points are used.)

The probability $p\left(s_{j}\right)$ is the prior probability that a data point is seabed type $s_{j}$. For the results presented here, we have used $p\left(s_{1}\right)=0.91$ and $p\left(s_{2}\right)=p\left(s_{3}\right)=p\left(s_{4}\right)=0.03$, which represents a strong (prior) belief that the seabed type will be flat $\left(s_{1}\right)$. As a result, for an area of seabed to be classified as rippled $\left(s_{2}\right)$, rocky $\left(s_{3}\right)$, or mixed $\left(s_{4}\right)$, particularly strong evidence that supports such a decision will be required. The particular priors used here are an approximation to the empirical distribution of the seabed types found in the training data. However, it should also be noted that as more views are used, the importance and influence of the particular priors chosen diminishes (cf., [19]).

Upon training one separate GMM for each of the four seabed types-flat, rippled, rocky, and mixed-each data point is evaluated using (11). These calculations result in the posterior probability that each data point belongs to each seabed type. Probabilistic seabed classification is based on these posterior probabilities.

\section{B. Visual Presentation of Results}

The posterior probability that each data point belongs to each seabed type is best displayed graphically in the form of posterior probability "maps." These posterior probability maps for each seabed type are generated according to the following convention. The higher the posterior probability for the given seabed type, the brighter the color. Thus, a purely black pixel corresponds to a posterior probability of zero.

If one were instead interested in a hard decision regarding the seabed type, the maximum a posteriori (MAP) probability-taken across seabed types-would be used for the classification. However, as noted previously, making hard decisions ignores both the full complexity of the seabed classification problem and the richness of information that the proposed approach provides.

The uncertainty in the classification decisions can be expressed by an associated "entropy map." Each entropy map shows the uncertainty of the classification decision-as quantified by the entropy of the posterior probabilities, calculated from (14) - for each data point. In these maps, white pixels correspond to high uncertainty, while black pixels correspond to low uncertainty. It should be noted that the range of possible entropy values for this application is from 0 bits to 2 bits (since there are $J=4$ seabed types).

In the results that follow in Section VII-B, the posterior probability maps for each seabed type, the maximum a posteriori classification results, and the associated entropy maps are all shown. To facilitate easy comparison among results, all figures use the same axis limits.

\section{EXPERIMENTAL RESULTS}

\section{A. Case Study}

As a prelude to the full set of experimental results, we first examine the results of the proposed approach on one particular data point. This case study will illuminate some subtle yet important points necessary for the proper interpretation of the full results.

The four views of the data point we consider here are shown in Fig. 8. The data point appears markedly different in each view because each view is at a unique orientation and at a different range to the sonar. It should also be noted that the image from the southeast-northwest survey, in Fig. 8(d), is blurry due to significant undesired vehicle motion (i.e., yaw, pitch, and roll) at the time of data collection. This example highlights the fact that exploiting multiple views can prevent the reliance on poor-quality single-view data for performing classification. 


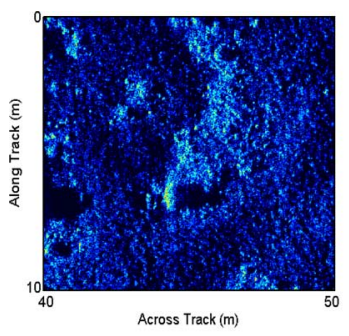

(a)

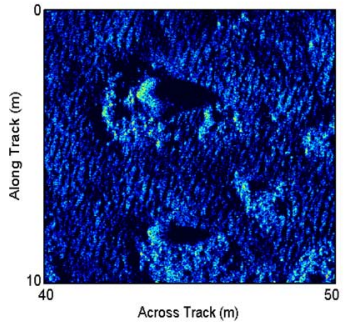

(c)

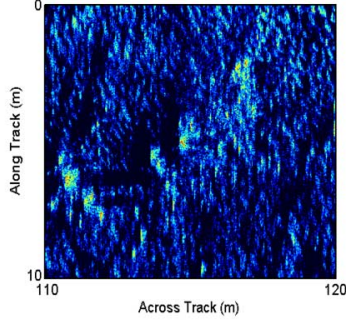

(b)

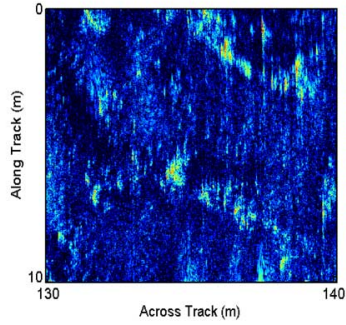

(d)
Fig. 8. SAS imagery of one data point as seen from the (a) north-south, (b) northeast-southwest, (c) east-west, and (d) southeast-northwest survey directions.

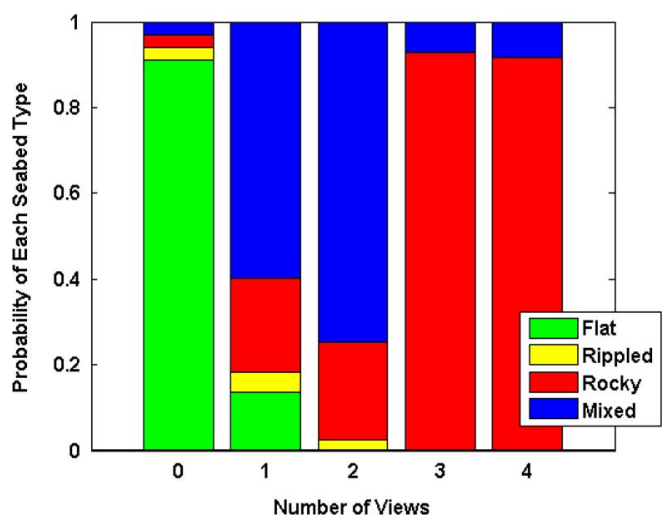

Fig. 9. Evolution of the posterior probabilities of belonging to each seabed type as additional views are acquired for the data point shown in Fig. 8. The probabilities for the case of no views are the prior probabilities.

This data point was ground-truthed as "rocky" seabed. However, from Fig. 8, it can be seen that the data point also contains characteristics of a "mixed" seabed. Thus, this example illustrates-as did Fig. 1 - that a data point may not belong to a single clear-cut seabed type.

As a result, it would be unreasonable to expect that the posterior probability of belonging to the "rocky" seabed type would approach unity. In turn, the entropy of the posterior probabilities of seabed type should not be expected to approach zero.

In this case study, we consider the resulting classification of the data point from using the proposed approach, assuming the views of the data point are acquired in the order shown in Fig. 8. The resulting evolution of the posterior probabilities of belonging to each seabed type as additional views are acquired for the data point is shown in Fig. 9.

If one were forced to make a hard classification decision regarding seabed type based on the MAP probability after only one or two views were obtained, the data point would be clas-
TABLE I

For the Data POINT SHOWn IN Fig. 8, THE (PRIOR OR POSTERIOR) PROBABILITY OF THE TRUE SEABED TYPE ("ROCKY") AND THE ENTROPy of the Probabilities of THE SEABED TyPe

\begin{tabular}{|c||c|c|}
\hline $\begin{array}{c}\text { NUMBER } \\
\text { OF VIEWS }\end{array}$ & $\begin{array}{c}\text { PROBABILITY OF } \\
\text { ROCKY SEABED }\end{array}$ & $\begin{array}{c}\text { ENTROPY } \\
\text { (BITS) }\end{array}$ \\
\hline \hline 0 & 0.0300 & 0.5791 \\
\hline 1 & 0.2188 & 1.5280 \\
\hline 2 & 0.2271 & 0.9459 \\
\hline 3 & 0.9280 & 0.3824 \\
\hline 4 & 0.9181 & 0.4089 \\
\hline
\end{tabular}

sified as "mixed" seabed. However, it can be seen readily from Fig. 9 that making a hard decision would ignore the richness of information that the probabilistic nature of the predictions provides. This is one reason why assessing classification performance based on hard decisions is of limited value.

A more appropriate quantity for evaluating classification performance is based on the probability of belonging to the true (i.e., ground-truthed) seabed type. This quantity, as well as the entropy of the (prior or posterior) probabilities of the seabed type, is shown in Table I for the data point under consideration.

The results in Table I illustrate how, with additional views, the entropy of the seabed predictions typically decreases and the posterior probability of the true seabed type generally increases. These trends are, in general, desirable. However, as noted previously, this particular data point contains characteristics of both "rocky" seabed and "mixed" seabed. As a result, desiring the posterior probability of the ground-truthed seabed type- - "rocky"- to reach unity (and the entropy to go to zero) is an unrealistic goal. Instead, a lower posterior probability of the true seabed type and a higher entropy (than the ersatz "ideal" case associated with zero entropy) is actually a more accurate reflection of the true nature of the data point.

These subtle points are important to keep in mind when assessing the ensuing set of full experimental results.

\section{B. Classification Results}

1) Qualitative Results: Experiments have been conducted for every possible combination of the four views of data (with one, two, three, and four survey directions considered at a time). Due to space constraints, we explicitly show the results for only the four single-view cases and the one four-view case.

Shown for each case are the posterior probability maps, the seabed classification based on the maximum a posteriori probabilities that would result if a hard classification decision were required, and the uncertainty in those classification decisions reflected via an associated entropy map. For the single-view data from the east-west survey, these results are shown in Figs. 10 and 11(a) and (b), respectively. For the single-view data from the north-south survey, these results are shown in Figs. 12 and 13(a) and (b), respectively. For the single-view data from the northeast-southwest survey, these results are shown in Figs. 14 and 15(a) and (b), respectively. For the single-view data from the southeast-northwest survey, these results are shown in Figs. 16 and 17 (a) and (b), respectively. For the multiview case in which the data from all four survey directions is used, the results are shown in Figs. 18 and 19(a) and (b), respectively. 


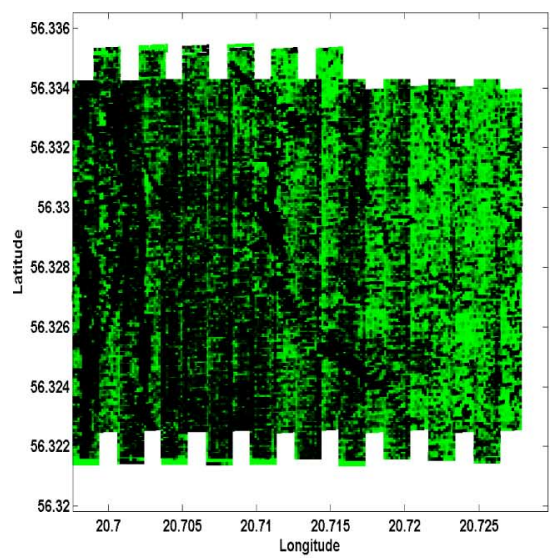

(a)

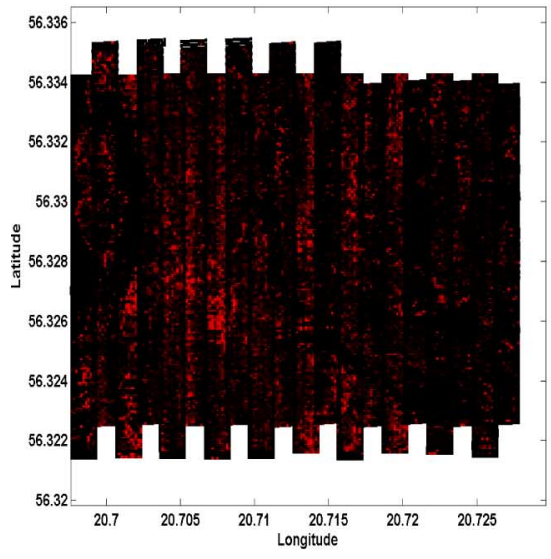

(c)

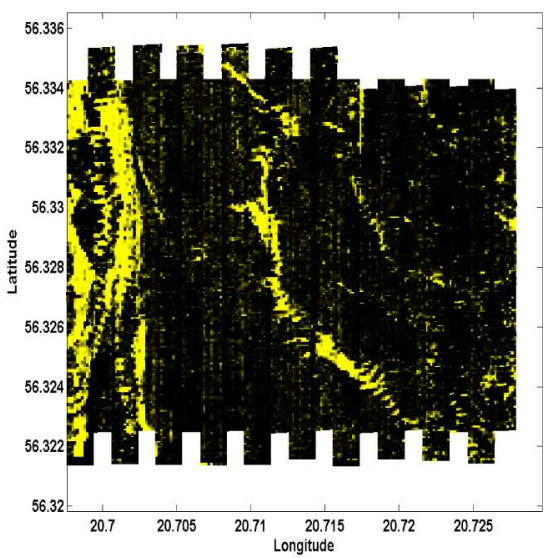

(b)

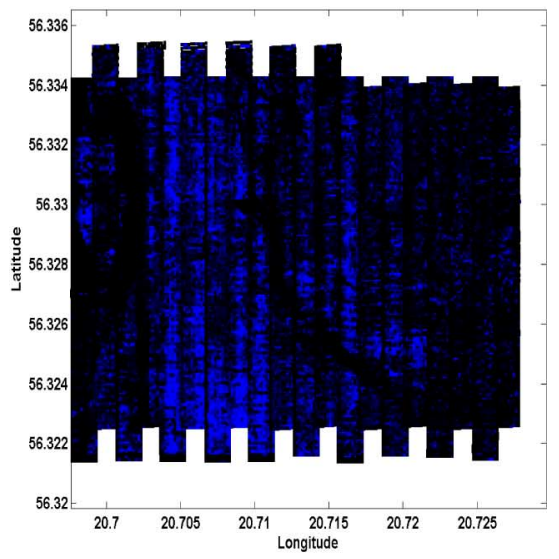

(d)

Fig. 12. Posterior probability maps from the north-south survey for (a) flat seabed, (b) rippled seabed, (c) rocky seabed, and (d) mixed seabed. The brighter the color, the higher the posterior probability for the given seabed type.

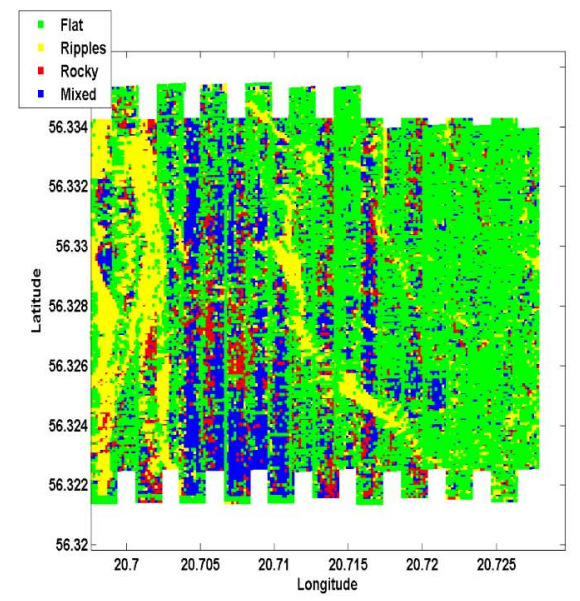

(a)

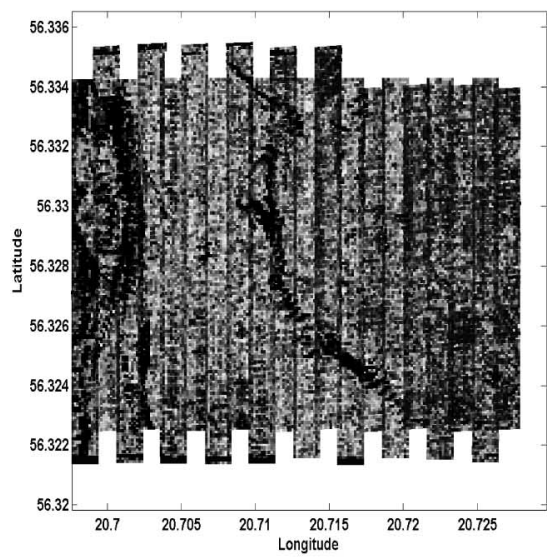

(b)

Fig. 13. From the north-south survey, (a) single-view seabed classification results based on the seabed type with the MAP probability, and (b) the entropy map associated with the classifications (where white denotes the highest uncertainty, and black denotes the lowest uncertainty).

2) Quantitative Results: In general, a higher posterior probability for the true seabed type is an indication of improved classification performance. However, as was noted in Section VII-A, an accurate reflection of the true nature of a data point would not necessarily be achieved when this posterior probability is very high.
Nevertheless, for every possible combination of the four views of data (with one, two, three, and four survey directions considered at a time), we show in Table II the mean posterior probability of the true seabed type (averaged over the data points). The results shown are for only those data points that 


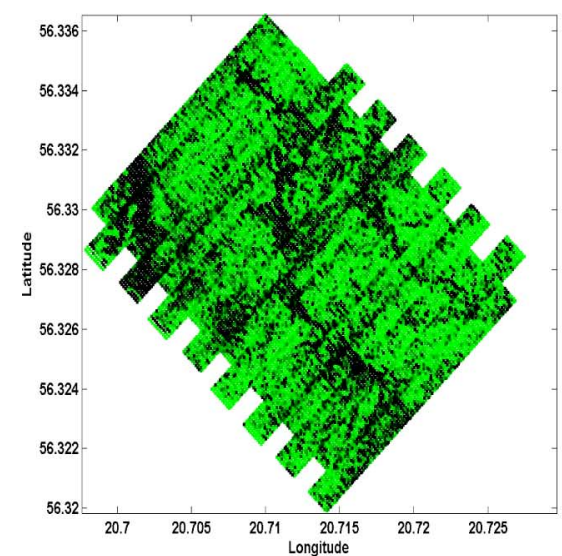

(a)

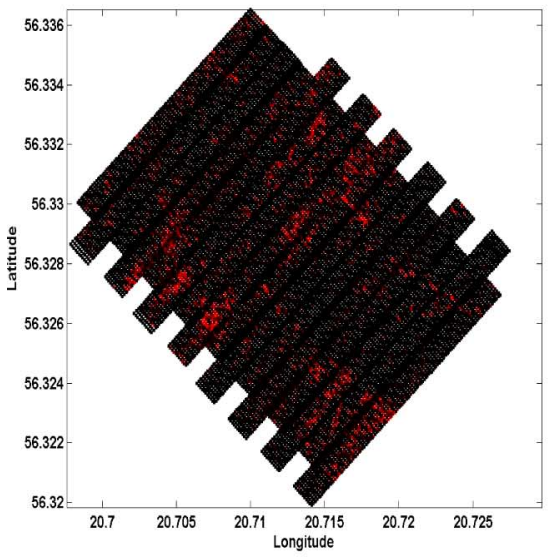

(c)

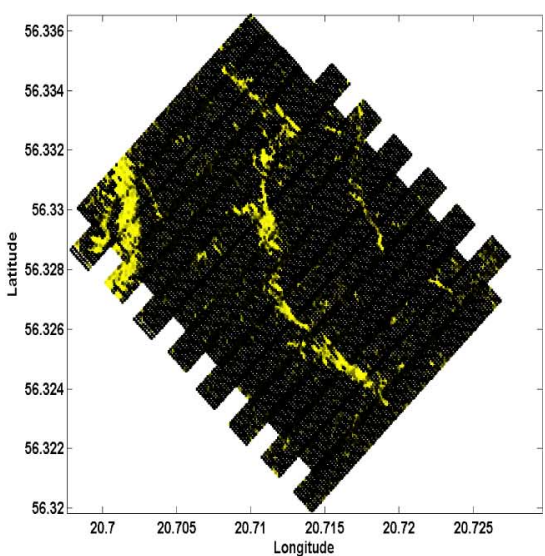

(b)

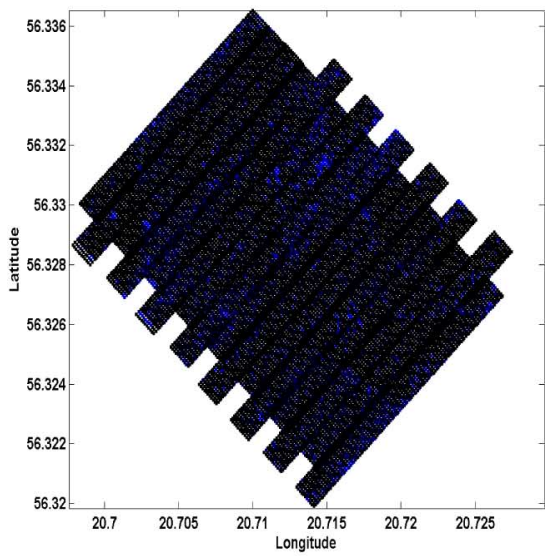

(d)

Fig. 14. Posterior probability maps from the northeast-southwest survey for (a) flat seabed, (b) rippled seabed, (c) rocky seabed, and (d) mixed seabed. The brighter the color, the higher the posterior probability for the given seabed type.

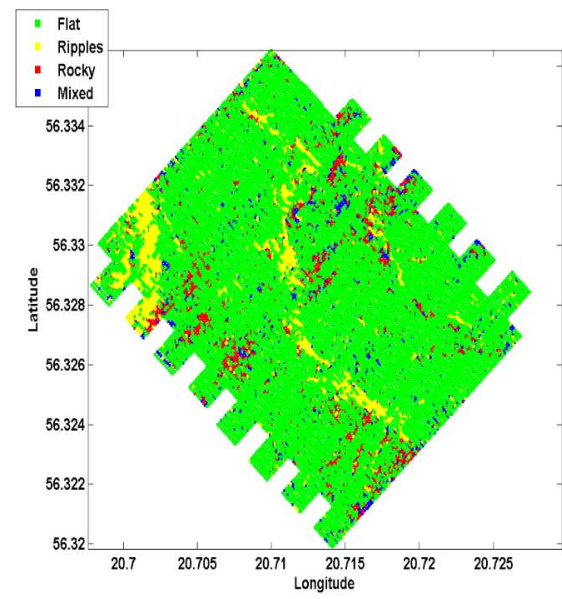

(a)

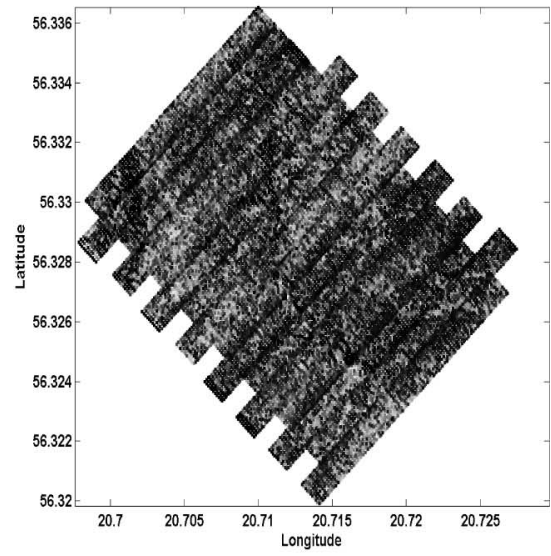

(b)

Fig. 15. From the northeast-southwest survey, (a) single-view seabed classification results based on the seabed type with the MAP probability, and (b) the entropy map associated with the classifications (where white denotes the highest uncertainty, and black denotes the lowest uncertainty).

were not used for training, but for which ground truth information is available.

From Table II, it can be observed that the inclusion of multiple views tended to cause the posterior probability of the true seabed types to converge to a "steady-state" value that reconciles all of the information from the disparate views.

Next, performance is assessed when hard classification decisions regarding seabed type are forced. As was stated in Section V-B, obtaining a seabed classification result that per- 


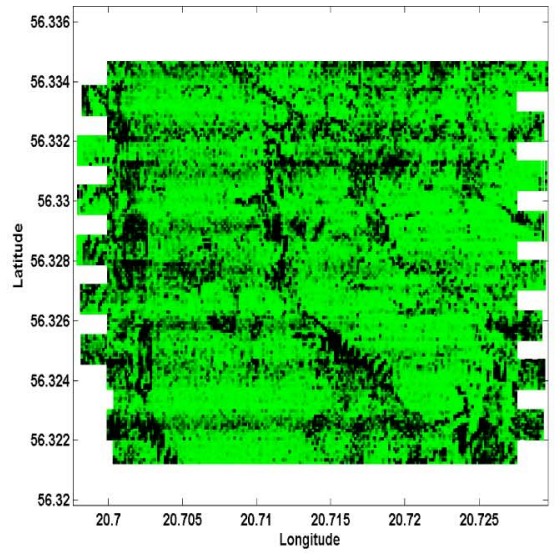

(a)

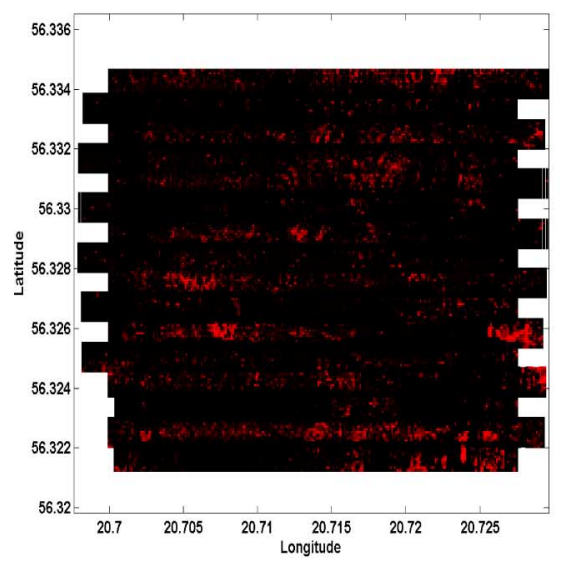

(c)

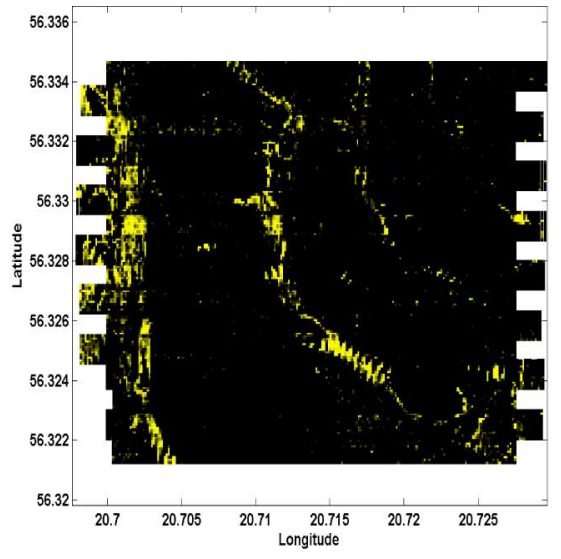

(b)

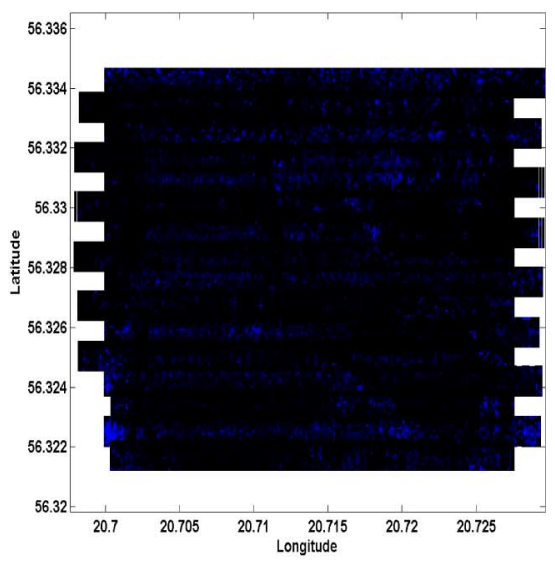

(d)

Fig. 10. Posterior probability maps from the east-west survey for (a) flat seabed, (b) rippled seabed, (c) rocky seabed, and (d) mixed seabed. The brighter the color, the higher the posterior probability for the given seabed type.

fectly matches the manual ground-truth is an unrealistic-and not completely desirable - goal. For one, it should be noted that the ground truth was created by examining the data from only one particular orientation. If viewed from a different orientation, it is reasonable to assume that the ground truth result would not be identical.

Nevertheless, to demonstrate in a different manner the benefit of using multiple views for seabed classification, we present confusion matrices for the classification results based on MAP probabilities. Each confusion matrix represents only those data points that were not used for training but for which ground truth information is available. Specifically, the $i j$ th entry in a confusion matrix expresses the fraction of times that the $i$ th seabed type was classified as the $j$ th seabed type.

We show four distinct confusion matrices, each of which corresponds to the case of using a different total number of views to perform classification. More specifically, each confusion matrix is the average of the confusion matrices from every possible combination of survey directions for a fixed number of total views. The average confusion matrices from using one view (averaged over the four possible cases), from using two views (averaged over the six possible combinations), from using three views (averaged over the four possible combinations), and from using four views (the one possible case) are shown in Tables III-VI, respectively.

As can be seen from Tables III-VI, classification performance improves, in general, as additional views are added. However, it should be reiterated that the probabilistic nature of the proposed approach provides more information than is exploited by a hard decision.

A large portion of the classification differences ("errors") in Tables III-VI can be attributed to imperfect ground truthing. For example, it can be argued that blocks ground-truthed as "rocky" seabed which often contained only a few rock-like objects would be more appropriately ground-truthed as "flat" or "mixed" seabed. Similarly, the difference between the "flat" and "mixed" seabed classes-typically only the absence or presence of shell deposits-is often difficult to judge. Moreover, blocks that contain sand ripples in only a portion of the block are also prone to be misclassified when a hard decision is demanded.

These situations highlight the fact that the classification of seabed type is rarely a simple clear-cut decision. It is precisely for these reasons that it is imperative that a probabilistic seabed classification algorithm be employed. 


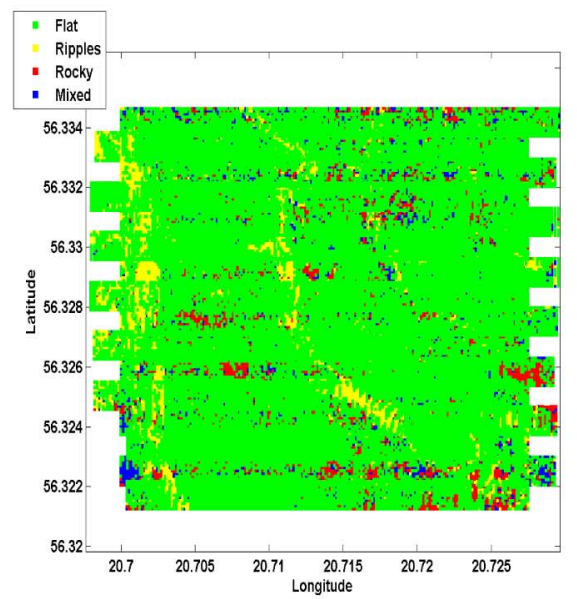

(a)

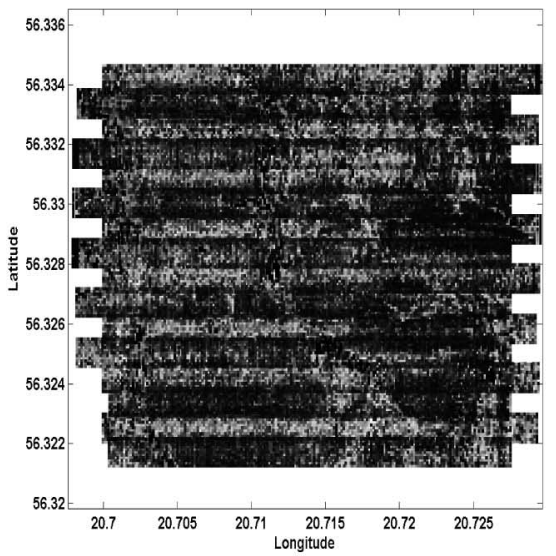

(b)

Fig. 11. From the east-west survey, (a) single-view seabed classification results based on the seabed type with the MAP probability, and (b) the entropy map associated with the classifications (where white denotes the highest uncertainty, and black denotes the lowest uncertainty).

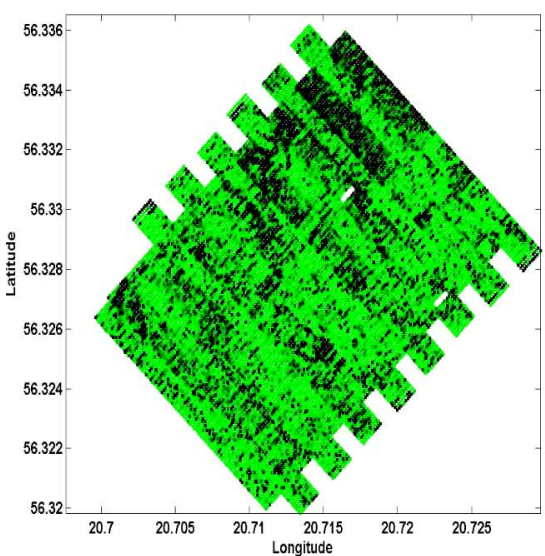

(a)

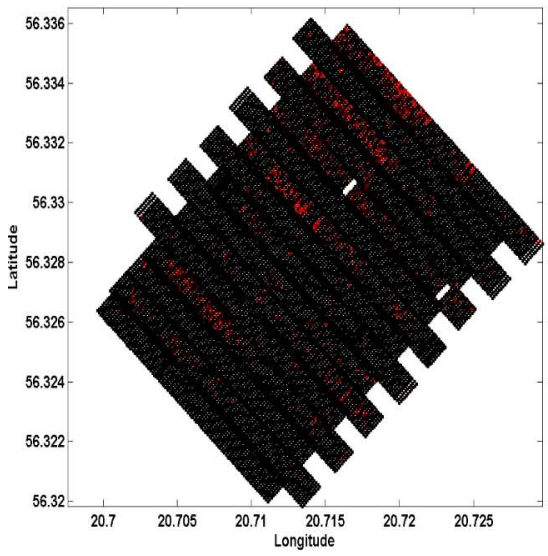

(c)

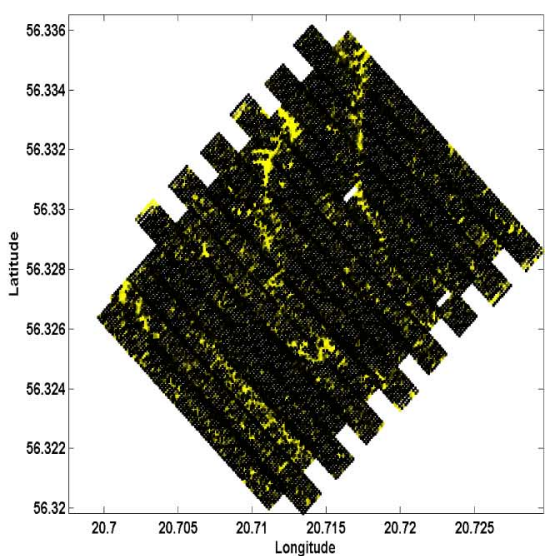

(b)

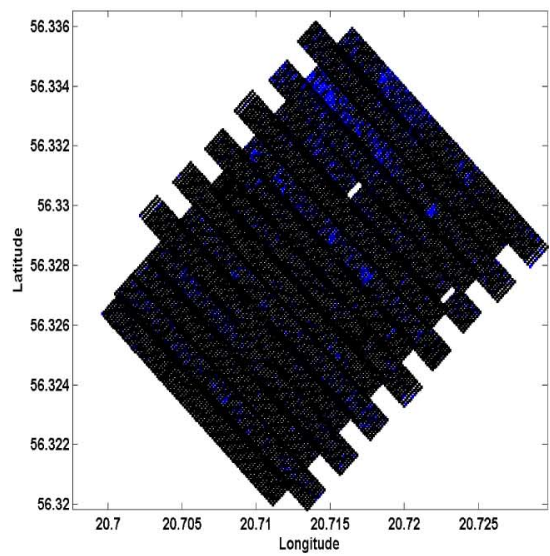

(d)

Fig. 16. Posterior probability maps from the southeast-northwest survey for (a) flat seabed, (b) rippled seabed, (c) rocky seabed, and (d) mixed seabed. The brighter the color, the higher the posterior probability for the given seabed type. 


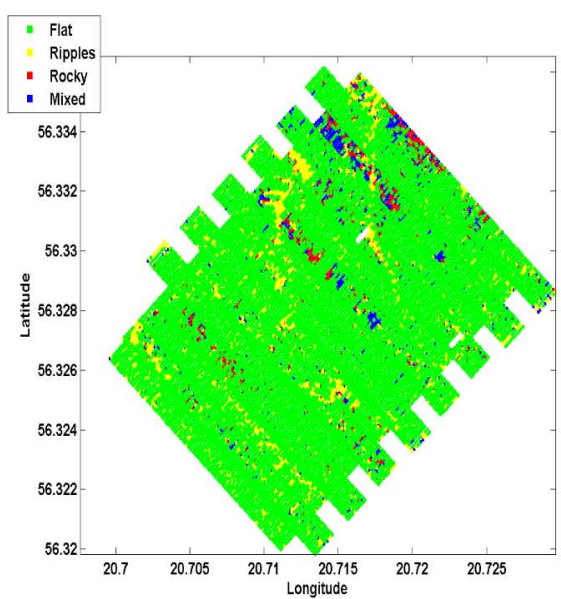

(a)

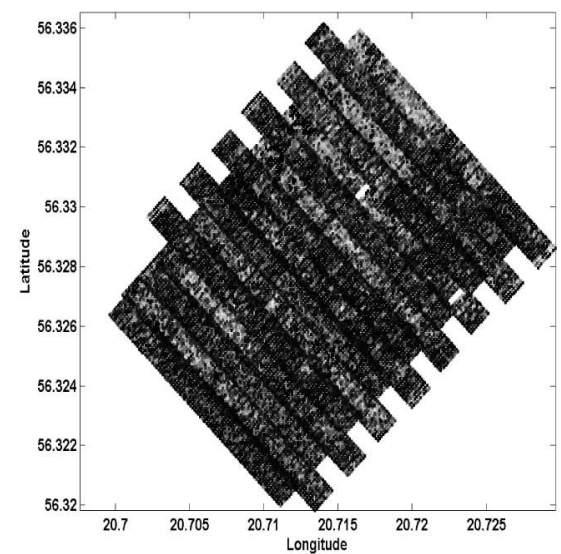

(b)

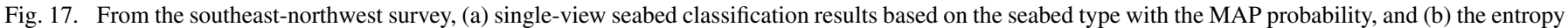
map associated with the classifications (where white denotes the highest uncertainty, and black denotes the lowest uncertainty).

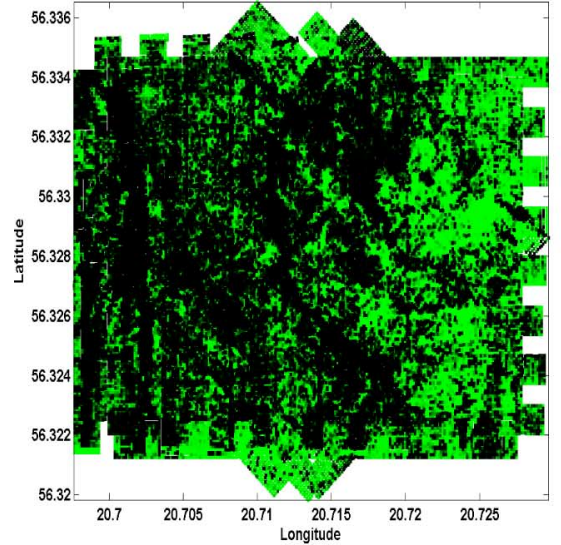

(a)

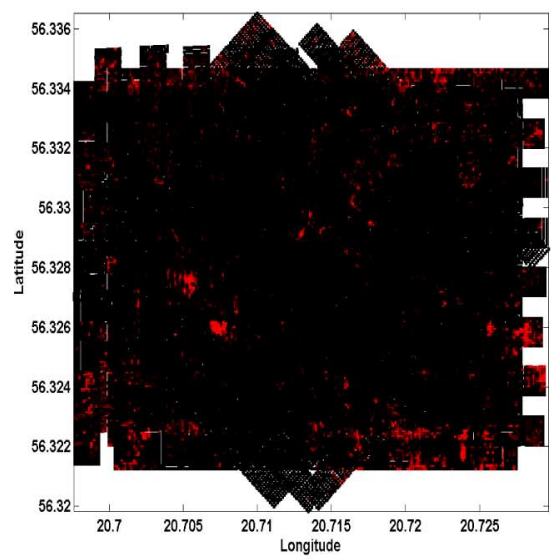

(c)

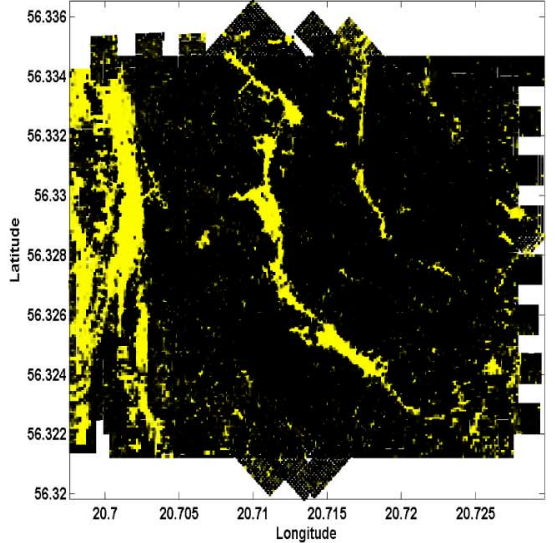

(b)

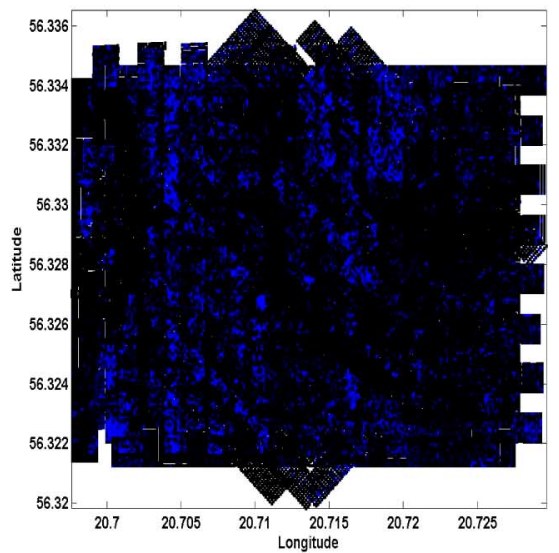

(d)

Fig. 18. Multiview posterior probability maps from the north-south, northeast-southwest, east-west, and southeast-northwest surveys for (a) flat seabed, (b) rippled seabed, (c) rocky seabed, and (d) mixed seabed. The brighter the color, the higher the posterior probability for the given seabed type.

Additionally, as will be shown in Section VII-C, the majority of the classification "errors" in Table VI are for data points whose maximum a posteriori classification was with low confidence (i.e., high uncertainty). 


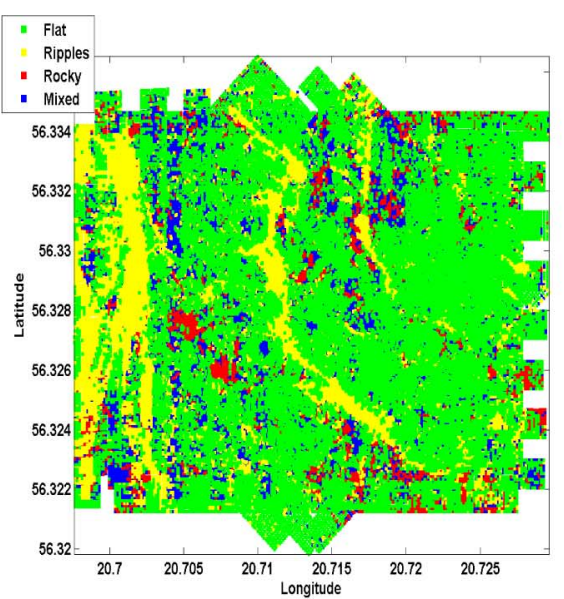

(a)

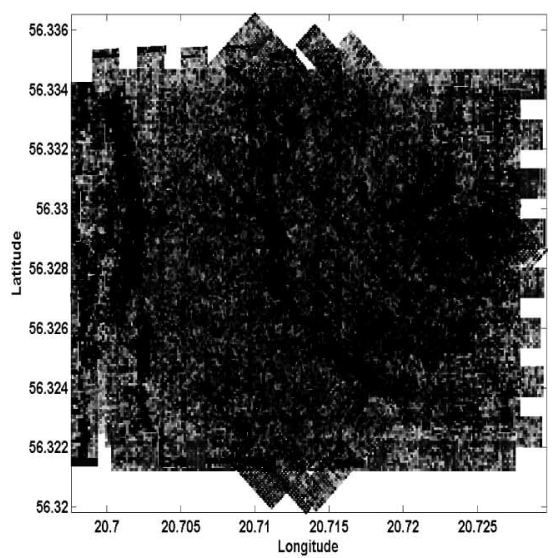

(b)

Fig. 19. From the north-south, northeast-southwest, east-west, and southeast-northwest surveys, (a) multiview seabed classification results based on the seabed type with the MAP probability, and (b) the entropy map associated with the classifications (where white denotes the highest uncertainty, and black denotes the lowest uncertainty).

TABLE II

Average Posterior Probability of the TRUe SEABed Type

\begin{tabular}{|c|c|c|}
\hline $\begin{array}{c}\text { NUMBER } \\
\text { OF ViEWS }\end{array}$ & SURVEY DiREctions & AVERAGE \\
PROBABILITY \\
\hline \hline 1 & N-S & 0.4286 \\
1 & NE-SW & 0.6216 \\
1 & E-W & 0.7001 \\
1 & SE-NW & 0.5946 \\
\hline 2 & N-S, NE-SW & 0.4457 \\
2 & N-S, E-W & 0.5186 \\
2 & N-S, SE-NW & 0.4669 \\
2 & NE-SW, E-W & 0.6828 \\
2 & NE-SW, SE-NW & 0.6099 \\
2 & E-W, SE-NW & 0.6775 \\
\hline 3 & N-S, NE-SW, E-W & 0.5209 \\
3 & N-S, NE-SW, SE-NW & 0.4891 \\
3 & N-S, E-W, SE-NW & 0.5559 \\
3 & NE-SW, E-W, SE-NW & 0.6799 \\
\hline 4 & N-S, NE-SW, E-W, SE-NW & 0.5617 \\
\hline
\end{tabular}

TABLE III

Average Confusion Matrix of the SEABEd Classification Results BASED ON THE MAP PRoBabiLITIES, From the Four ONE-VIEW CASES

\begin{tabular}{|c||c|c|c|c|}
\hline \multicolumn{1}{|c||}{ GROUND TRUTH } & \multicolumn{4}{c|}{ PREDICTED SEABED TYPE } \\
SEABED TYPE & FLAT & RIPPLED & ROCKY & MIXED \\
\hline \hline FLAT & $\mathbf{0 . 7 8 5 4}$ & 0.0564 & 0.0390 & 0.1192 \\
\hline RIPPLED & 0.3832 & $\mathbf{0 . 5 2 5 0}$ & 0.0543 & 0.0374 \\
\hline ROCKY & 0.5971 & 0.0520 & $\mathbf{0 . 2 1 5 3}$ & 0.1356 \\
\hline MIXED & 0.6723 & 0.0614 & 0.1305 & $\mathbf{0 . 1 3 5 8}$ \\
\hline
\end{tabular}

\section{Entropy Results}

The results of the proposed multiview seabed classification algorithm were presented in Section VII-B. Comparisons of the various maximum a posteriori classification results with the manual ground-truth in Fig. 6 suggest that the results are reasonable for providing a global assessment of the highly-complex area.

However, for active learning in particular, the posterior probabilities for each seabed type, and in turn the entropy of the predictions, are of more interest. By calculating the entropy of the
TABLE IV

Average Confusion Matrix of the SEabed Classification RESUlTS BASED ON THE MAP PROBABILITIES, FROM THE SIX TWO-VIEW SURVEY COMBINATIONS

\begin{tabular}{|c||c|c|c|c|}
\hline \multicolumn{1}{|c||}{ GROUND TRUTH } & \multicolumn{4}{c|}{ PREDICTED SEABED TYPE } \\
SEABED TYPE & FLAT & RIPPLED & ROCKY & MIXED \\
\hline \hline FLAT & $\mathbf{0 . 6 7 6 0}$ & 0.1047 & 0.0357 & 0.1836 \\
\hline RIPPLED & 0.1988 & $\mathbf{0 . 7 1 5 3}$ & 0.0387 & 0.0472 \\
\hline ROCKY & 0.4020 & 0.0900 & $\mathbf{0 . 2 4 7 5}$ & 0.2606 \\
\hline MIXED & 0.4988 & 0.1089 & 0.1405 & $\mathbf{0 . 2 5 1 8}$ \\
\hline
\end{tabular}

TABLE V

AVERage Confusion Matrix OF THE SEABEd Classification RESULTS BASED ON THE MAP PROBABILITIES,

FROM THE FOUR THREE-VIEW SURVEY COMBINATIONS

\begin{tabular}{|c||c|c|c|c|}
\hline \multicolumn{1}{|c||}{ GROUND TRUTH } & \multicolumn{4}{c|}{ PREDICTED SEABED TYPE } \\
SEABED TYPE & FLAT & RIPPLED & ROCKY & MIXED \\
\hline \hline FLAT & $\mathbf{0 . 6 1 8 0}$ & 0.1154 & 0.0271 & 0.2395 \\
\hline RIPPLED & 0.1018 & $\mathbf{0 . 8 1 9 3}$ & 0.0275 & 0.0514 \\
\hline ROCKY & 0.2925 & 0.0895 & $\mathbf{0 . 2 4 2 5}$ & 0.3755 \\
\hline MIXED & 0.3906 & 0.1202 & 0.1291 & $\mathbf{0 . 3 6 0 1}$ \\
\hline
\end{tabular}

TABLE VI

Confusion Matrix of the Four-VIEW Seabed Classification Results BASED ON THE MAP PROBABILITIES

\begin{tabular}{|c||c|c|c|c|}
\hline \multicolumn{1}{|c||}{ GROUND TRUTH } & \multicolumn{4}{c|}{ PREDICTED SEABED TYPE } \\
SEABED TYPE & FLAT & RIPPLED & ROCKY & MIXED \\
\hline \hline FLAT & $\mathbf{0 . 5 9 1 3}$ & 0.1094 & 0.0188 & 0.2805 \\
\hline RIPPLED & 0.0632 & $\mathbf{0 . 8 6 5 5}$ & 0.0175 & 0.0539 \\
\hline ROCKY & 0.2184 & 0.0871 & $\mathbf{0 . 2 2 7 1}$ & 0.4674 \\
\hline MIXED & 0.3194 & 0.1218 & 0.1107 & $\mathbf{0 . 4 4 8 0}$ \\
\hline
\end{tabular}

seabed classification for each data point (i.e., area of seabed) using (14), and then averaging across all data points in the entire data set (that were not used for training), the average classification uncertainty in seabed type can be established. This average entropy quantity is computed for every possible survey combination, with the results presented in Table VII.

From the results in Table VII, it can be seen that the average entropy decreases as additional views are used. Specifically, the 
TABLE VII

AVERAGE ENTROPY OF SEABED ClasSIFICATION

\begin{tabular}{|c|c|c|}
\hline $\begin{array}{c}\text { NUMBER } \\
\text { OF } \\
\text { VIEWS }\end{array}$ & SURVEY DIRECTIONS & $\begin{array}{c}\text { AVERAGE } \\
\text { ENTROPY } \\
\text { (BITS) }\end{array}$ \\
\hline \hline 1 & N-S & 0.8066 \\
1 & NE-SW & 0.6502 \\
1 & E-W & 0.5269 \\
1 & SE-NW & 0.4659 \\
\hline 2 & N-S, NE-SW & 0.6394 \\
2 & N-S, E-W & 0.5678 \\
2 & N-S, SE-NW & 0.5699 \\
2 & NE-SW, E-W & 0.4933 \\
2 & NE-SW, SE-NW & 0.4469 \\
2 & E-W, SE-NW & 0.3782 \\
\hline 3 & N-S, NE-SW, E-W & 0.4780 \\
3 & N-S, NE-SW, SE-NW & 0.4797 \\
3 & N-S, E-W, SE-NW & 0.4237 \\
3 & NE-SW, E-W, SE-NW & 0.3543 \\
\hline 4 & N-S, NE-SW, E-W, SE-NW & 0.3718 \\
\hline
\end{tabular}

average entropy of the multiview case was always less than the highest average entropy from among the single views considered. However, the use of an additional view did not always decrease the classification uncertainty. Instead, the inclusion of multiple views appeared to cause the average entropy to converge to a "steady-state" value, which may reflect the true uncertainty inherent in the data. (Recall that a given area of seabed is rarely a single, clear-cut type.)

For the four-view case, the average entropy of blocks that were classified correctly based on the maximum a posteriori classification was 0.2732 bits. The average entropy of blocks that were classified incorrectly based on the maximum a posteriori classification was 0.4835 bits. Thus, the confidence in the predictions was significantly lower for the cases that were not correct. This suggests that the active-learning approach proposed to improve classification-based on obtaining additional views of the areas of seabed with the highest entropy values - is sensible.

1) Active-Learning Results: To demonstrate the benefit of the active-learning method concretely, we conduct the following experiment (using only those data points that were not used for training, but for which ground truth information is available). It is assumed that data from a single survey direction is possessed initially. The entropy of the posterior probabilities for each seabed type can then be computed for each data point.

For the proposed active-learning approach, additional views should be acquired first for the data point with the highest entropy (i.e., uncertainty). More generally, the order of data points for which additional views should be acquired are from those with the highest entropy to those with the lowest entropy. An alternative approach to active learning would be to randomly select the order of data points to acquire additional views for.

We compare the results of the active-learning approach with the results of randomly selecting which data point to acquire additional views for, in terms of the mean posterior probability of the true seabed type (as in Table II). Specifically, this quantity is shown in Fig. 20 as a function of the fraction of data points for which the remaining views were acquired. Results are shown when each of the four survey directions are assumed to be the

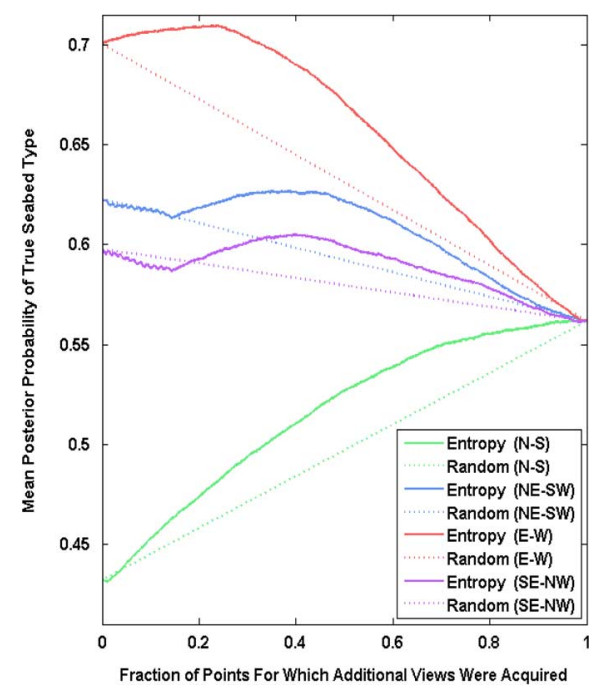

Fig. 20. Active-learning comparison of acquiring additional views randomly versus based on the data points with the highest seabed-classification entropy. Shown is the mean posterior probability of belonging to each data point's true seabed type, as a function of the fraction of data points whose remaining views were acquired. The legend indicates the method by which the data points to acquire additional views for were selected, with the initial survey-direction view indicated in parentheses.

TABLE VIII

AREa Under CuRVES (IN Fig. 20) OF MEAN POSTERIOR PROBability of TRUE SEABED TYPES USING ENTROPY-BASED CRITERION AND RANDOM SELECTION (MEAN \pm ONE StaNdard DEVIaTION, From 100 Trials)

\begin{tabular}{|c||c|c|}
\hline \multicolumn{1}{|c||}{ INITIAL } & \multicolumn{2}{c|}{ AREA UNDER CURVE } \\
VIEW & ENTROPY & RANDOM \\
\hline \hline N-S & 0.5158 & $0.4970 \pm 0.0009$ \\
\hline NE-SW & 0.6061 & $0.5922 \pm 0.0011$ \\
\hline E-W & 0.6566 & $0.6309 \pm 0.0011$ \\
\hline SE-NW & 0.5888 & $0.5796 \pm 0.0012$ \\
\hline
\end{tabular}

initial view. When additional views are acquired for a data point, it is assumed that all (i.e., up to three) remaining views of the data point are acquired.

For the case of randomly selecting which data points to acquire additional views for, 100 independent trials are conducted; each trial uses a unique, random acquisition order. The results presented in Fig. 20 for this "random" approach are the mean results over the 100 trials. (Error bars, representing one standard deviation about this mean value, were very tight, as is reflected in Table VIII).

The entropy-based active-learning results in Fig. 20 suggest that acquiring additional views of data points whose seabed predictions have high entropy tends to increase the posterior probability of the true seabed type. The converse also tends to hold, as evidenced by the fact that the latter portions of the curves in Fig. 20 associated with the entropy-based approach have decreasing slopes.

In practice, resources are limited, so the improvement of classification (e.g., in terms of the posterior probability of the true seabed type) as quickly as possible is desirable. A measure of the success of each approach, therefore, can be quantified by calculating the areas under the curves in Fig. 20. Active-learning performance using this quantity is shown in Table VIII, where higher values for this "area" are preferred. 
It should be noted that in practice, path-planning algorithms should also be considered when active learning is conducted, since additional data (i.e., views) would be obtained for areas of seabed, not just for a single point. Nevertheless, these experimental results suggest that the entropy-based criterion is a sensible way to conduct active learning for improving seabed classification. Moreover, the results show that the proposed method is superior to a strategy that randomly selects which additional views to acquire.

\section{DISCUSSION}

This paper addresses the task of seabed classification when multiple views are available from SAS imagery. In addition, we consider the task of active learning, which has never before been addressed in the context of this problem.

The focus of this paper was not on feature development for the seabed classification problem. Instead, the main contribution of this paper is the development of the basic framework for combining data from multiple views to perform seabed classification. As such, this paper is a proof-of-concept for the fusion of data to achieve multiview seabed classification. Because of its general nature, the proposed framework can easily be used with the feature sets developed by other researchers.

Employing multiple views to perform seabed classification allows one to make predictions based on a greater pool of information. As a result, the likelihood of making a prediction based on single-view data that is of poor quality — due, for example, to undesired vehicle motion or difficult sea conditions during data collection-is mitigated. In addition, the Bayesian framework of the proposed approach provides a natural way to leverage $a$ priori information about the seabed classification task with the observed (multiview) data.

Importantly, the proposed approach also results in probabilistic predictions of the seabed type. This probabilistic nature is particularly valuable because the complexity of an area of seabed rarely warrants a hard classification decision (cf., Fig. 1). Moreover, the probabilistic predictions allow for an elegantly simple, yet rigorous, approach for performing active learning to improve classification.

Previous approaches [8]-[10] to the seabed classification problem with multiple views were ill-suited for active learning because they did not address the problem of multiview fusion at the data-level. That is, their choice of decision-level fusion - fusing classifier outputs-precluded the possibility of performing principled active learning.

It should be noted that no smoothing of the final classification result is performed in our proposed approach. Other seabed classification methods [9], [10] will smooth the result, with Markov random fields, because they are attempting pixel-level classification. In our paper, because each data point represents a $10 \mathrm{~m} \times 10 \mathrm{~m}$ area of seabed, adjacent data points frequently belong to different seabed types; therefore, smoothing is not only unnecessary, but would also be detrimental to the classification result. (Another drawback of attempting to perform pixel-level classification is the unreasonable expectation of extremely precise navigational information from the sonar-equipped vehicle.) The data on which the proposed approach was applied was not a toy problem with artificially created textures patched together to simulate a seabed. Rather, the enormous area considered was real data with challenging, ill-defined regions of different seabed types. Multiview seabed classification has never before been attempted in the literature on a scale of this magnitude. Moreover, this paper represents the first to address the problem of seabed classification using high-resolution synthetic aperture sonar imagery, rather than simple side-scan sonar imagery.

Finally, a modification to the calculation of the joint likelihood that took into account the orientation dependence of sand ripples was presented in Section III-B. It was observed (in results not shown here) that the effects of changing the value of $\delta$ are minimal, with the effect of the modification greater on data points that possess few views.

\section{CONCLUSION}

A Bayesian data fusion approach for seabed classification using multiview synthetic aperture sonar (SAS) imagery has been proposed. Wavelet-based features were employed in the experiments presented, but the classification approach is general in that any type of features can be used. The distribution of the features extracted from the data points, conditioned on the seabed type, is modeled as a mixture of Gaussians. The end result of this formulation for a given data point is the posterior probability that it belongs to each seabed type.

In the derivation of the algorithm, each assumption that was invoked was both discussed and justified. The principled approach also explicitly accounts for the fact that rippled seabed can appear to be flat seabed at certain sonar-ripple orientations.

The elegant simplicity of the proposed seabed classification approach allows all available information to be exploited, while not requiring that every data point possess the same number of views. Attractively, a lack of a view for one data point does not adversely affect the predictions for other data points.

An approach to perform active learning based on the entropy of the classification predictions was also outlined. Experimental results on real multiview SAS imagery suggested that this natural criterion is suitable for determining how to best improve classification performance. This approach can be exploited in the adaptation of the route of an AUV during a mission.

This paper was a proof-of-concept for a new Bayesian multiview seabed classification framework. The emphasis and focus was not on the specific set of features that were employed. As a result, the classification errors not attributable to imperfect ground truthing were likely due to poor discriminating power (among seabed types) of the features used. However, this basic multiview classification framework can now be exploited in the future by other researchers working on the development of more sophisticated features.

\section{ACKNOWLEDGMENT}

The author would like to thank J. Groen of NURC for providing the detection algorithm code used to generate Fig. 7. The author would also like to thank the anonymous reviewers for helpful comments that led to a much improved manuscript.

\section{REFERENCES}

[1] Y. Petillot, S. Reed, and E. Coiras, "A framework for evaluating underwater mine detection and classification algorithms using augmented reality," presented at the UDT Conf., 2006. 
[2] D. MacKay, "Information-based objective functions for active data selection," Neural Comput., vol. 4, no. 4, pp. 590-604, Jul. 1992.

[3] Z. Reut, N. Pace, and M. Heaton, "Computer classification of seabeds by sonar," Nature, no. 314, pp. 426-428, 1985.

[4] N. Pace and H. Gao, "Swathe seabed classification," IEEE J. Ocean Eng., vol. 13, no. 2, pp. 83-90, Apr. 1988.

[5] B. Dasarathy and E. Holder, "Image characterizations based on join gray-level run-length distributions," Pattern Recognit. Lett., vol. 12, no. 8, pp. 497-502, 1991.

[6] D. Tamsett, "Seabed characterisation and classification from the power spectra of sidescan sonar data," Marine Geophys. Res., vol. 15, no. 1, pp. 43-64, 1993.

[7] D. Carmichael, L. Linnett, S. Clarke, and B. Calder, "Seabed classification through multifractal analysis of sidescan sonar imagery," IEE Radar, Sonar, Navig., vol. 143, no. 3, pp. 140-148, June 1996.

[8] D. Kerneis and B. Zerr, "Multisensor fusion for seabed classification," in Proc. MTS/IEEE OCEANS, 2005, pp. 815-820.

[9] S. Reed, I. T. Ruiz, C. Capus, and Y. Petillot, "The fusion of large scale classified sidescan sonar image mosaics," IEEE Trans. Image Process., vol. 15, no. 7, pp. 2049-2060, Jul. 2006.

[10] E. Coiras, V. Myers, and B. Evans, "Reliable seabed characterization for MCM operations," presented at the MTS/IEEE OCEANS, Vancouver, BC, Canada, 2007.

[11] A. Dempster, N. Laird, and D. Rubin, "Maximum likelihood from incomplete data via the EM algorithm," J. Roy. Statist. Soc. B, vol. 39, pp. 1-38, 1977.

[12] N. Nasios and A. Bors, "Variational expectation-maximization training for Gaussian networks," in Proc. IEEE Workshop on Neural Networks for Signal Processing, 2003, pp. 339-348.

[13] M. Beal and Z. Ghahramani, "The variational Bayesian EM algorithm for incomplete data: Application to scoring graphical model structures," Bayesian Statist., vol. 7, pp. 453-464, 2003.
[14] M. Beal, "Variational Algorithms for Approximate Bayesian Inference," Ph.D. dissertation, Gatsby Computational Neuroscience Unit, University College London, , 2003.

[15] V. Fedorov, Theory of Optimal Experiments. New York: Academic, 1972.

[16] T. Cover and J. Thomas, Elements of Information Theory. New York: Wiley, 1991.

[17] S. Mallat, A Wavelet Tour of Signal Processing. New York: Academic, 1999

[18] J. Fawcett, A. Crawford, D. Hopkin, V. Myers, and B. Zerr, ComputerAided Detection of Targets From the CITADEL Trial Klein Sonar Data, Defence R \& D Canada-Atlantic, Tech. Rep. DRDC Atlantic TM 2006$115,2006$.

[19] R. Duda, P. Hart, and D. Stork, Pattern Classification, 2nd ed. New York: Wiley, 2001.

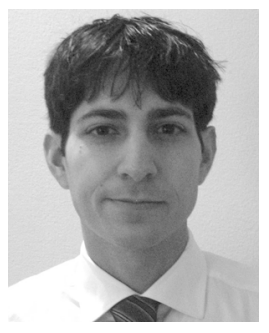

David P. Williams received the B.S.E. (magna cum laude), M.S., and Ph.D. degrees in electrical engineering from Duke University in 2002, 2003, and 2006, respectively.

Upon graduating, he joined Signal Innovations Group, Durham, NC. Since August 2007, he has been with the Mine Countermeasures Group, NATO Undersea Research Centre, La Spezia, Italy. His research interests lie in the fields of machine learning, pattern recognition, and statistical signal processing.

Dr. Williams was the recipient of a James B. Duke Graduate Fellowship (from Duke) and a National Defense Science and Engineering Graduate Fellowship (from the Department of Defense). 